\title{
On the Kerr-AdS/CFT correspondence
}

\author{
Julián Barragán Amado, ${ }^{a, b}$ Bruno Carneiro da Cunha ${ }^{a}$ and Elisabetta Pallante ${ }^{b}$ \\ ${ }^{a}$ Departamento de Física, Universidade Federal de Pernambuco, \\ 50670-901, Recife, Pernambuco, Brazil \\ ${ }^{b}$ Van Swinderen Institute for Particle Physics and Gravity, \\ University of Groningen, Groningen, The Nederlands \\ E-mail: j.j.barragan.amado@rug.nl, bcunha@df.ufpe.br, epallante@rug.nl
}

ABSTRACT: We review the relation between four-dimensional global conformal blocks and field propagation in $\mathrm{AdS}_{5}$. Following the standard argument that marginal perturbations should backreact in the geometry, we turn to the study of scalar fields in the generic Kerr- $\mathrm{AdS}_{5}$ geometry. On one hand, the result for scattering coefficients can be obtained exactly using the isomonodromy technique, giving exact expressions in terms of $c=1$ chiral conformal blocks. On the other hand, one can use the analogy between the scalar field equations to the Level 2 null field Ward identity in two dimensional Liouville field theory to write approximate expressions for the same coefficients in terms of semi-classical chiral Liouville conformal blocks. Surprisingly, the conformal block thus constructed has a well-behaved interpretation in terms of Liouville vertex operators.

KeYwords: AdS-CFT Correspondence, Black Holes, Black Holes in String Theory

ARXIV EPRINT: 1702.01016 


\section{Contents}

1 Introduction 1

2 CFTs and scalar fields in $\mathrm{AdS}_{5} \quad 2$

2.1 The Kerr-AdS $\mathrm{S}_{5}$ background 5

$\begin{array}{lll}2.2 & \text { Kerr-anti de Sitter wave equation } & 7\end{array}$

2.3 Isomonodromy and formal solutions 9

3 Semi-classical conformal blocks and the near-extremal case 13

$\begin{array}{lll}3.1 & \text { Entropy and Liouville momenta } & 16\end{array}$

$\begin{array}{lll}4 & \text { Discussion } & 21\end{array}$

\section{Introduction}

The gauge/gravity correspondence has opened up a new avenue to study non-perturbative phenomena in field theories at conformal fixed points and some patterns of conformal symmetry breaking, using (semi)-classical gravitational analogues. In 20 years since its inception, a plethora of different applications has sprung, from exact results pertaining to integrable sectors in supersymmetric Yang-Mills (SYM) to phenomenological model building. In most of those, the role of black hole backgrounds as an analogue of a thermal state is paramount - see [1] for a review. In fact, perturbations of the thermal state, or analogously perturbations of the black hole, are fundamental to the understanding of the lowest-lying spectrum of the underlying theory.

In many of those models, the perturbation comes in the guise of a semi-classical scalar field in a classical black hole background. It serves as a mock-up model of true interactions in large-N $\mathcal{N}=4 \mathrm{SYM}$ and some deformations of it $[2,3]$, like $\mathcal{N}=2 \mathrm{SYM}$ with generic matter multiplets in the adjoint and/or fundamental. In general relativity, it also tests linear stability of solutions [4], relaxation times [5], as well as relation with the Conformal Field Theory (CFT) bootstrap, represented by Liouville field theory [6]. Most of the applications, however, either focus on low dimension, with the Bañados-Teitelboim-Zanelli (BTZ) black hole [7] serving as a ubiquitous background, or on higher-dimensional solutions without rotation - a serious drawback for many interesting physical phenomena, like superradiance, the zero temperature moduli and the fate of the inner horizon.

One powerful method to study rotating black holes in any dimension is the monodromy method, introduced in this context by $[8,9]$. The method has deep ties to integrable systems and the Riemann-Hilbert problem in complex analysis, relating scattering coefficients to monodromies of a flat holomorphic connection associated to the radial differential equation obtained after separation of variables. Recently, one of the authors helped uncover 
the relation between scattering coefficients of some Kerr-de Sitter [10, 11] black holes in four dimensions to exact $c=1$ conformal blocks using deformations of the flat holomorphic connection - isomonodromy deformations. These conformal blocks were in itself computed exactly [12] in terms of the Painlevé VI $\tau$-function, capitalizing on recent developments in gauge/gravity correspondence in the form of the Alday-Gaiotto-Tachikawa (AGT) conjecture $[13,14]$.

The relation between scattering amplitudes of black holes in any dimension and twodimensional conformal blocks has been alluded to by many authors and used to obtain bulk information from the boundary dynamics, see [15-19]. The purpose of the present work is to review the relation of semi-classical (global) conformal blocks in four dimensions to propagating (scalar) fields in five-dimensional anti-de Sitter (AdS), a fact outlined by [20] and $[18,21,22]$ revisiting work by Ferrara and collaborators in the 70's [23-25]. The construction does not rely on supersymmetry. We will also be interested in the exact relations between two-dimensional conformal blocks and scattering of scalar fields in a Kerr-AdS background in five dimensions, whose greybody factors are also given in terms of the Painlevé VI $\tau$-function. Due to this mathematical fact, the background displays a hidden conformal symmetry - albeit not directly connected to the one presented in [26]. In an appropriately defined semiclassical limit, these blocks are described by Liouville field theory and the corresponding vertex operators are matched.

The paper is structured as follows. In section 2, we review the work of [20] and give the $\mathrm{AdS}_{5}$ interpretation of four-dimensional CFT correlation functions — see also [25] - and close by discussing the modifications allowed by asymptotically $\mathrm{AdS}_{5}$ spaces. In Subection 2.1, we turn to the five-dimensional Kerr-AdS black hole, and write the scalar perturbation equation of motion in terms of two Heun ordinary differential equations (Fuchsian differential equations with 4 regular singular points). In subsection 2.3, we present formal solutions for the black hole normal modes in terms of the Painlevé VI $\tau$-function, thus relating them to $c=1$ exact conformal blocks. In section 3, we point out that the near extremal limit should be well described by semi-classical (global) conformal blocks, which in turn are well described by semi-classical Liouville field theory. We use this analogy to construct an equivalence between the solutions for the scalar field in Kerr-AdS 5 to Liouville 4-point correlators, with insertions associated to the singular points of the differential equation. We point out that, while the singular points of the angular equation, as well as the insertions associated to the point at infinity and the "unphysical" point $r_{0}$ in the radial equation are associated to usual Liouville vertex operators, the insertions associated to the inner and outer horizons are on the Seiberg bound line. We close by discussing the usefulness and relevance of the results for model building and outline the prospect of future work.

\section{CFTs and scalar fields in $\mathrm{AdS}_{5}$}

We review a few well-known facts about scalar fields in AdS space. The reader unfamiliar with the conformal bootstrap in generic dimensions may find the review in [27] useful. We will begin the discussion by considering the conformal block of a generic dual conformal field 


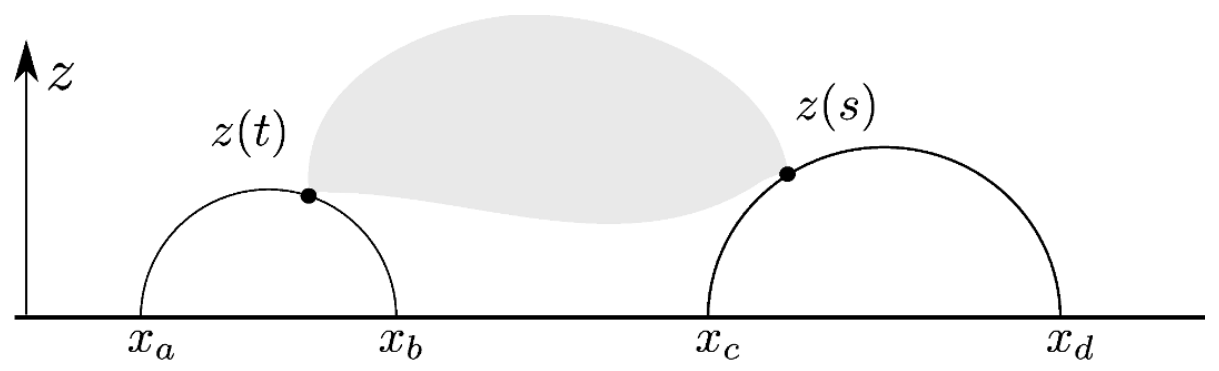

Figure 1. The boundary classical conformal block in the AdS representation. While the points at spatial infinity $(z=0)$ are linked by geodesics (the semicircles), the points at the geodesics are linked by the scalar field propagator. The mass $\mu$ of the propagator is related to the intermediate channel conformal weight $\Delta$ by $(2.6)$.

theory, defined by the projection of the four point function of primary (spinless) operators onto the conformal descendants of (the equally spinless) $\mathcal{O}$,

$$
Q\left(x_{a}, x_{b}, x_{c}, x_{d}\right)=\left\langle\mathcal{O}_{a}\left(x_{a}\right) \mathcal{O}_{b}\left(x_{b}\right) P_{\mathcal{O}} \mathcal{O}_{c}\left(x_{c}\right) \mathcal{O}_{d}\left(x_{d}\right)\right\rangle
$$

It has been verified that, under generic conditions $[18,20,22,24], Q$ is given by the expression:

$$
\begin{aligned}
Q= & K C_{a b \Delta} C_{\Delta c d}\left(\left(x_{a b}\right)^{2}\right)^{-\frac{\Delta_{a}+\Delta_{b}}{2}}\left(\left(x_{c d}\right)^{2}\right)^{-\frac{\Delta_{c}+\Delta_{d}}{2}} \\
& \times \int_{0}^{\infty} \frac{d t}{t^{\frac{\Delta_{a}-\Delta_{b}}{2}}+1} \int_{0}^{\infty} \frac{d s}{s^{\frac{\Delta_{c}-\Delta_{d}}{2}+1}}\left\langle\Phi_{\Delta}(z(t), x(t)) \Phi_{\Delta}(z(s), x(s))\right\rangle_{0},
\end{aligned}
$$

where $t=(1-u) / u$ and $s=(1-v) / v$, with $u$ and $v$ spanning the geodesics as above, see also figure 1 . The constant $K$ is a product of beta functions:

$$
K=\frac{4^{d-\Delta} \Gamma(\Delta)^{2}}{\Gamma\left(\frac{1}{2}(a \Delta b)\right) \Gamma\left(\frac{1}{2}(b \Delta a)\right) \Gamma\left(\frac{1}{2}(c \Delta d)\right) \Gamma\left(\frac{1}{2}(d \Delta c)\right)} .
$$

The variables $\Phi_{\Delta}$ are constructed from the intermediate primary fields $\mathcal{O}_{\Delta}$ from a boundary-to-bulk procedure - again we refer to the literature cited above for details. The function between brackets

$$
\left\langle\Phi_{\Delta}\left(z_{1}, x_{1}\right) \Phi_{\Delta}\left(z_{2}, x_{2}\right)\right\rangle_{0}=G_{n}\left(z_{1}, x_{1} ; z_{2}, x_{2}\right)
$$

denotes a particular integral transformation of the two-point function of $\mathcal{O}_{\Delta}$ in a conformally invariant "vacuum-state" in the CFT.

The expression for $Q$ has a natural interpretation as the propagation of a scalar field in a $\mathrm{AdS}_{d+1}$ background, with metric given by

$$
d s^{2}=\frac{d z^{2}+\eta_{\mu \nu} d x^{\mu} d x^{\nu}}{z^{2}} .
$$

where the mass is related to the conformal dimension of the intermediate channel by

$$
\Delta=d / 2+\sqrt{d^{2} / 4+\mu^{2}} .
$$


In the Poincaré metric, the semicircles in figure 1 are geodesics, and the bulk-to-bulk propagator between the internal points is given by the free scalar field in $\operatorname{AdS}_{d+1}$ :

$$
G_{n}(\sigma)=2^{-\Delta} \sigma^{-\Delta} F_{1}\left(\frac{1}{2} \Delta, \frac{1}{2} \Delta+\frac{1}{2} ; \Delta-\frac{d-2}{2} ; \sigma^{-2}\right)
$$

with

$$
\sigma=2 z-1=\frac{z_{1}^{2}+z_{2}^{2}+\eta_{\mu \nu}\left(x_{1}-x_{2}\right)^{\mu}\left(x_{1}-x_{2}\right)^{\nu}}{2 z_{1} z_{2}} .
$$

related to the geodesic distance by $\sigma=\cosh (\ell)$. We define $z$ for future reference.

The results above establish a geometric interpretation of conformal systems in any dimension, and open up the possibility of studying conformal symmetry breaking mechanisms through the dynamics of geometry, a tool widely used in general relativity. This point of view is supported via the Maldacena conjecture, where some (relevant) perturbations of the CFT vacuum state $|0\rangle$ can be modelled through the deformation of the background AdS space-time. Formulas like the boundary-to-bulk propagator as well as the Witten diagram (2.2) can be suitably modified by changing the path of integration and the bulk-bulk propagator, in order to accommodate for the change of geometry. If the space-time is only asymptotically anti-de Sitter, then the exact formulas above will only hold when the separations between points are small, i.e., in the UV limit. Asymptotically AdS spaces will then correspond to UV conformal fixed points in the boundary theory.

Even beyond SUSY and exact backgrounds, there is some evidence to trust that quantum corrections can be geometrized in a way similar to above, at least for a class of conformal field theories and for some patterns of conformal symmetry breaking. In particular, this should be true for boundary field theories where the decoupling from the lowest-lying low-energy spectrum of the theory happens as described in [28]. In this case, for instance, if we take the "cluster-decomposition" limit of (2.2) where

$$
x_{a} \simeq x_{b}, \quad x_{c} \simeq x_{d}, \quad\left|x_{c}-x_{a}\right| \gg 1,
$$

we can think of the AdS propagator as giving the would-be meson-meson potential. Another important question is if zero-temperature QCD realizes such decoupling. The results put forward in [29] for large-N Yang-Mills say that the logarithmic corrections to conformal symmetry induced by asymptotic freedom in the scalar glueball two-point function differ from the UV behaviour of AdS/CFT realizations. At the same time, confinement and asymptotic freedom are two aspects of one single phenomenon of conformal symmetry breaking. The latter may be illustrated by a naive geometric "hard wall" construction, for which the discrete would-be meson mass spectrum is given by

$$
\frac{p_{n}^{2}}{\Lambda^{2}}=-x_{\nu, n},
$$

where $\Lambda=1 / z_{0}$ is an infrared cutoff and $x_{\nu, n}$ is the n-th zero of the regular Bessel function $J_{\nu}(x)$, has nevertheless phenomenologically appealing features and could still serve as a description of the deconfined phase of QCD, in the presence of mass (temperature) perturbations. In other words, the successes in describing quark-gluon plasma resonances 
via AdS/CFT genuinely depend on the deconfined and near-conformal nature of the hightemperature system, where the conformally-invariant Coulomb potential is corrected by temperature and mass perturbations.

Certainly, the geometrization described in this section points to the fact that there is a class of broken-CFTs that are described by an asymptotically AdS space-time. Admittedly, the conditions presented here are much weaker than having, for instance, a generic background where quantum corrections are under control. Such class of broken-CFTs should at least include the deconfined region of (supersymmetric) non-Abelian gauge theories described in the classical tests of AdS/CFT, as well as many examples of low-dimensional condensed matter systems.

In the next section, we consider rotating black hole solutions in AdS as a tool to describe thermal field theories, and to deviate from pure AdS towards the infrared. The relevant quantities on the geometric side of the correspondence are basically restricted to the black hole mass, angular momenta and whichever monopole charges we include in the theory. By analyzing this restricted and still tractable space of parameters, one could hope to learn more about a broad spectrum of dual CFTs.

\subsection{The Kerr-AdS 5 background}

We pick the simplest asymptotically AdS background in five dimensions, Kerr-AdS ${ }_{5}$. From the argument of the last section, its purported dual field theory will display a (trivial) conformal fixed point in the UV, with no mass gap. Scalar field perturbations around this background will give the would-be scalar meson spectrum of the boundary theory, as per the correspondence built in the preceding section, eventually allowing us to describe a class of breaking patterns of the boundary CFT in the UV. On the other hand, the IR behavior will be radically different from pure AdS, with the horizon now playing the role of a thermal background. One notes two new features of the analysis presented here. Firstly, the holographic interpretation of the space-time charges is not unique, it will depend on the conformal structure we choose for the asymptotic metric. The choice consists, from a four dimensional perspective, to consider the CFT living either on a spatial plane or a sphere. Analyzing the three elements of the Cartan subalgebra of $\operatorname{SO}(4,2)$, we see that in both choices the time-translation is kept, so the energy is the same in either of them. The angular momenta, though, can be thought of as values from translational momenta or one component of the translational momentum, say $p_{z}$, and the angular momentum parallel to it. We will take the latter interpretation. Secondly, there is a non-trivial moduli of parameters even at zero temperature: in five dimensions there is a one-parameter family of zero temperature black holes which can be thought to represent non-trivial conformal fixed points in the IR of the dual theory.

Also, some thermodynamical comments are in order. Because of the rotation, the Killing vector associated with the outer horizon becomes space-like sufficiently far from the source, so that any thermal radiation would have to move faster than light to maintain equilibrium. The analysis of [30] has shown that thermodynamical equilibrium is possible for Kerr-AdS black holes in a region of the parameter space, and there exist phase transition(s) to a region where the boundary manifold rotates faster than the speed of light 
at least in one direction. A duality is also inferred [30] between the Kerr-AdS black hole at critical angular velocities and the CFT (in one dimension less) at the boundary of AdS space. The extremal Kerr-AdS ${ }_{5}$ black hole has been further thermodynamically studied in [31], revealing another class of holographic duality. The equality of thermodynamical quantities (entropy) suggests a duality of the extremal Kerr-AdS black hole (in any dimension) and a chiral 2d CFT for each rotation (see also [32]). Thus, for rotating and asymptotically AdS black holes in five dimensions, one expects two types of holographic duality: one with a four-dimensional CFT on the rotating manifold at the boundary of AdS, using AdS/CFT correspondence, the other with chiral two-dimensional CFTs (one for each rotation) in the extremal black hole limit, using Kerr/CFT correspondence in the near horizon region. As we will see, the analysis presented here will point to a third option.

The Kerr- $\mathrm{AdS}_{5}$ metric is [30]

$$
\begin{aligned}
d s^{2}= & -\frac{\Delta_{r}}{\rho^{2}}\left(d t-\frac{a_{1} \sin ^{2} \theta}{1-a_{1}^{2}} d \phi-\frac{a_{2} \cos ^{2} \theta}{1-a_{2}^{2}} d \psi\right)^{2}+\frac{\Delta_{\theta} \sin ^{2} \theta}{\rho^{2}}\left(a_{1} d t-\frac{\left(r^{2}+a_{1}^{2}\right)}{1-a_{1}^{2}} d \phi\right)^{2} \\
& +\frac{1+r^{2}}{r^{2} \rho^{2}}\left(a_{1} a_{2} d t-\frac{b\left(r^{2}+a_{1}^{2}\right) \sin ^{2} \theta}{1-a_{1}^{2}} d \phi-\frac{a\left(r^{2}+a_{2}^{2}\right) \cos ^{2} \theta}{1-a_{2}^{2}} d \psi\right)^{2} \\
& +\frac{\Delta_{\theta} \cos ^{2} \theta}{\rho^{2}}\left(a_{2} d t-\frac{\left(r^{2}+a_{2}^{2}\right)}{1-a_{2}^{2}} d \psi\right)^{2}+\frac{\rho^{2}}{\Delta_{r}} d r^{2}+\frac{\rho^{2}}{\Delta_{\theta}} d \theta^{2}
\end{aligned}
$$

where

$$
\begin{aligned}
& \Delta_{r}=\frac{1}{r^{2}}\left(r^{2}+a_{1}^{2}\right)\left(r^{2}+a_{2}^{2}\right)\left(1+r^{2}\right)-2 M \\
& \Delta_{\theta}=1-a_{1}^{2} \cos ^{2} \theta-a_{2}^{2} \sin ^{2} \theta, \quad \rho^{2}=r^{2}+a_{1}^{2} \cos ^{2} \theta+a_{2}^{2} \sin ^{2} \theta
\end{aligned}
$$

and $a_{1}$ and $a_{2}$ are two independent rotation parameters. This metric satisfies $R_{\mu \nu}=-4 g_{\mu \nu}$, and asymptotically approaches AdS space with radius of curvature $\ell_{\text {AdS }}=1$. The mass relative to the $\mathrm{AdS}_{5}$ vacuum solution and the angular momenta are

$$
\mathcal{M}=\frac{3 \pi M}{4\left(1-a_{1}^{2}\right)\left(1-a_{2}^{2}\right)}, \quad \mathcal{J}_{\phi}=\frac{\pi M a_{1}}{2\left(1-a_{1}^{2}\right)^{2}}, \quad \mathcal{J}_{\psi}=\frac{\pi M a_{2}}{2\left(1-a_{2}^{2}\right)^{2}}
$$

The horizons of the black hole are obtained from the equation $\Delta_{r}=0$, which can be reduced to a cubic equation by making the substitution $x=r^{2}$. For real $a_{1}, a_{2}$ and positive $M$ there are two real roots to this equation, $r_{+}^{2}$ and $r_{-}^{2}$. The largest of these roots, $r_{+}^{2}>r_{-}^{2}$, corresponds to the outer event horizon [33]. Note that this metric possesses three commuting Killing vectors,

$$
\xi_{k}=\frac{\partial}{\partial t}+\Omega_{a}\left(r_{k}\right) \frac{\partial}{\partial \phi}+\Omega_{b}\left(r_{k}\right) \frac{\partial}{\partial \psi}
$$

such that they are null at each respective horizon and has the time translational and rotational (bi-azimuthal) isometries. This entails to the constants $\Omega_{k} \equiv \Omega\left(r_{k}\right)$ being the angular velocities of each horizon and one can interpret these quantities as angular velocities 
in two independent orthogonal 2-planes of rotation. The temperature and angular velocities of each horizon for an observer following $\xi_{k}$ orbits, with $k=+,-, 0$, are given by

$$
\begin{aligned}
\Omega_{k, 1} & =\frac{a_{1}\left(1-a_{1}^{2}\right)}{r_{k}^{2}+a_{1}^{2}} \quad \Omega_{k, 2}=\frac{a_{2}\left(1-a_{2}^{2}\right)}{r_{k}^{2}+a_{2}^{2}} \\
T_{k} & =\frac{r_{k}^{2} \Delta^{\prime}\left(r_{k}\right)}{4 \pi\left(r_{k}^{2}+a_{1}^{2}\right)\left(r_{k}^{2}+a_{2}^{2}\right)}=\frac{r_{k}}{2 \pi} \frac{\left(r_{k}^{2}-r_{i}^{2}\right)\left(r_{k}^{2}-r_{j}^{2}\right)}{\left(r_{k}^{2}+a_{1}^{2}\right)\left(r_{k}^{2}+a_{2}^{2}\right)} .
\end{aligned}
$$

We note for future reference that for reasonable values of $M, a_{1}$ and $a_{2}, r_{+}$and $r_{-}$are real and positive, while $r_{0}^{2}=-r_{-}^{2}-r_{+}^{2}-a_{1}^{2}-a_{2}^{2}-1$ is negative. By the same token, $T_{+}$is positive, $T_{-}$is negative and $T_{0}$ is purely imaginary. In the extremal case $r_{+}=r_{-}, T_{ \pm}$goes to zero linearly with $r_{+}^{2}-r_{-}^{2}$ while $T_{0}$ is finite. We also note that (2.11) reduces to the global five-dimensional AdS metric when $M=a_{1}=a_{2}=0$ and that the metric displays a singularity hidden by an event horizon when $T_{+} \geq T_{-} \geq 0$.

\subsection{Kerr-anti de Sitter wave equation}

One of the most interesting properties of Kerr-(A)dS black hole (2.11), with arbitrary rotation parameters in five dimensions, is the separability of the Hamilton-Jacobi equation for a particle, and also the separability of the Klein-Gordon (KG) equation for a scalar field in this background [34]. We consider now the KG equation with mass $\mu$ together with the Ansatz $\Phi=\Pi(r) \Theta(\theta) e^{-i \omega t+i m_{1} \phi+i m_{2} \psi}$ where $m_{1}, m_{2} \in \mathbb{Z}$. This mode solution results in two decoupled ordinary differential equations for radial and angular functions. The angular equation is given by

$$
\begin{aligned}
& \frac{1}{\sin \theta \cos \theta} \frac{d}{d \theta}\left(\sin \theta \cos \theta \Delta_{\theta} \frac{d \Theta(\theta)}{d \theta}\right)-\left[\omega^{2}+\frac{\left(1-a_{1}^{2}\right) m_{1}^{2}}{\sin ^{2} \theta}+\frac{\left(1-a_{2}^{2}\right) m_{2}^{2}}{\cos ^{2} \theta}\right. \\
& \left.-\frac{\left(1-a_{1}^{2}\right)\left(1-a_{2}^{2}\right)}{\Delta_{\theta}}\left(\omega+m_{1} a_{1}+m_{2} a_{2}\right)^{2}+\mu^{2}\left(a_{1}^{2} \cos ^{2} \theta+a_{2}^{2} \sin ^{2} \theta\right)\right] \Theta(\theta)=-\lambda \Theta(\theta),
\end{aligned}
$$

where $\lambda$ is the separation constant. For more details, see [35], although we will present the necessary ingredients to bring the angular equation to the canonical form of a Heun equation. Thus, we use the transformation $u=\sin ^{2} \theta$, under which the four regular singular points are located at

$$
u=0, \quad u=1, \quad u=u_{0}=\frac{1-a_{1}^{2}}{a_{2}^{2}-a_{1}^{2}}, \quad u=\infty,
$$

and the critical exponents are defined as the asymptotic behavior of the function near the singular points $S(u) \simeq\left(u-u_{i}\right)^{\alpha_{i}}$ or $S(u) \simeq u^{-\alpha_{\infty}}$ for the point at infinity

$$
\alpha_{0}= \pm \frac{m_{1}}{2}, \quad \alpha_{1}= \pm \frac{m_{2}}{2}, \quad \alpha_{u_{0}}= \pm \frac{1}{2}\left(\omega+a_{1} m_{1}+a_{2} m_{2}\right), \quad \alpha_{\infty}=1 \pm \sqrt{1+\frac{\mu^{2}}{4}} .
$$

The sign choice is immaterial and we will take the positive solution as standard. Let us now define $\beta=\omega+a_{1} m_{1}+a_{2} m_{2}$. Performing the change of variables

$$
\Theta(u)=u^{-m_{1} / 2}(u-1)^{-m_{2} / 2}\left(u-u_{0}\right)^{-\beta / 2} S(u)
$$


we bring the angular equation to the canonical Heun form

$$
\frac{d^{2} S}{d u^{2}}+\left(\frac{1-m_{1}}{u}+\frac{1-m_{2}}{u-1}+\frac{1-\beta}{u-u_{0}}\right) \frac{d S}{d u}+\left(\frac{q_{1} q_{2}}{u(u-1)}+\frac{u_{0}\left(u_{0}-1\right) Q_{0}}{u(u-1)\left(u-u_{0}\right)}\right) S=0
$$

with the accessory parameters given by

$$
\begin{aligned}
q_{1} q_{2}= & \frac{1}{4}\left(\beta+m_{1}+m_{2}-2\right)^{2}-1-\frac{1}{4} \mu^{2} \\
4 u_{0}\left(u_{0}-1\right) Q_{0}= & \frac{\lambda-\omega^{2}-\mu^{2}}{a_{2}^{2}-a_{1}^{2}}+\left(u_{0}-1\right)\left(\left(m_{1}+\beta-1\right)^{2}-m_{2}^{2}-1\right) \\
& +u_{0}\left(\left(m_{2}+\beta-1\right)^{2}-m_{1}^{2}-1\right) .
\end{aligned}
$$

One notes that (2.20) has the same AdS spheroidal harmonics form as the problem in four dimensions.

The radial equation can be written in the form

$$
\begin{gathered}
\frac{1}{r \Pi(r)} \frac{d}{d r}\left(r \Delta_{r} \frac{d \Pi(r)}{d r}\right)-\left[\lambda+\mu^{2} r^{2}+\frac{1}{r^{2}}\left(a_{1} a_{2} \omega-a_{2}\left(1-a_{1}^{2}\right) m_{1}-a_{1}\left(1-a_{2}^{2}\right) m_{2}\right)^{2}\right] \\
+\frac{\left(r^{2}+a_{1}^{2}\right)^{2}\left(r^{2}+a_{2}^{2}\right)^{2}}{r^{4} \Delta_{r}}\left(\omega-\frac{m_{1} a_{1}\left(1-a_{1}^{2}\right)}{r^{2}+a_{1}^{2}}-\frac{m_{2} a_{2}\left(1-a_{2}^{2}\right)}{r^{2}+a_{2}^{2}}\right)^{2}=0 .
\end{gathered}
$$

This equation again has four regular singular points, located at the roots of $r^{2} \Delta_{r}$ and infinity

$$
r^{2}=r_{-}^{2}, \quad r^{2}=r_{+}^{2}, \quad r^{2}=r_{0}^{2}, \quad r^{2}=\infty,
$$

with critical exponents given in terms of the temperatures and angular velocities by

$$
\theta_{k}= \pm \frac{i}{2 \pi}\left(\frac{\omega-m_{1} \Omega_{k, 1}-m_{2} \Omega_{k, 2}}{T_{k}}\right), \quad \theta_{\infty}=\frac{\Delta}{2}, \frac{d-\Delta}{2}=1 \pm \sqrt{1+\frac{\mu^{2}}{4}}
$$

where $k=0,+,-$. As with the angular equation, the choice of root is immaterial and we will choose the positive sign to define the $\theta_{k}$. Note that $\theta_{\infty}$ is the scaling dimension from the CFT interpretation as in the analysis of last section. To bring this equation to the canonical Heun form, we perform the change of variables

$$
z=\frac{r^{2}-r_{-}^{2}}{r_{+}^{2}-r_{-}^{2}}
$$

and write it in terms of

$$
\Pi(z)=z^{-\theta_{-} / 2}(z-1)^{-\theta_{+} / 2}\left(z-z_{0}\right)^{-\theta_{0} / 2} R(z),
$$

where $z_{0}=\left(r_{0}^{2}-r_{-}^{2}\right) /\left(r_{+}^{2}-r_{-}^{2}\right)$. After some uneventful algebraic manipulations, we arrive at the equation for $R(z)$

$$
\frac{d^{2} R}{d z^{2}}+\left[\frac{1-\theta_{-}}{z}+\frac{1-\theta_{+}}{z-1}+\frac{1-\theta_{0}}{z-z_{0}}\right] \frac{d R}{d z}+\left(\frac{\kappa_{0} \kappa_{1}}{z(z-1)}+\frac{z_{0}\left(z_{0}-1\right) K_{0}}{z(z-1)\left(z-z_{0}\right)}\right) R(z)=0,
$$


where $z_{0}$ is given as above, and

$$
\begin{aligned}
\kappa_{0} \kappa_{1}= & \frac{1}{4}\left(\theta_{-}+\theta_{+}+\theta_{0}-2\right)^{2}-1-\frac{\mu^{2}}{4} \\
4 z_{0}\left(z_{0}-1\right) K_{0}= & -\frac{\lambda+\mu^{2} r_{0}^{2}-\omega^{2}}{r_{+}^{2}-r_{-}^{2}}+\left(z_{0}-1\right)\left[\left(\theta_{0}+\theta_{-}-1\right)^{2}-\theta_{+}^{2}-1\right] \\
& +z_{0}\left[\left(\theta_{0}+\theta_{+}-1\right)^{2}-\theta_{-}^{2}-1\right]
\end{aligned}
$$

Both equations (2.20) and (2.28) can be solved by usual Frobenius methods in terms of Heun series. We are, however, interested in solutions which satisfy

$$
S(u)= \begin{cases}1+\mathcal{O}(u), & u \rightarrow 0, \\ 1+\mathcal{O}(u-1), & u \rightarrow 1,\end{cases}
$$

which will set a quantization condition for the separation constant $\lambda$. Note that $u_{0}$ in $(2.20)$ is always greater than one for the $a_{2}<1$ case, below the extremal limit. The condition that $R(z)$ corresponds to a purely ingoing wave at the outer horizon $z=1$ and normalizable at infinity is

$$
R(z)= \begin{cases}1+\mathcal{O}(z-1), & z \rightarrow 1, \\ z^{-\Delta / 2-\left(\theta_{+}+\theta_{-}+\theta_{0}\right) / 2}\left(1+\mathcal{O}\left(z^{-1}\right)\right), & z \rightarrow \infty\end{cases}
$$

with $\Delta=2+\sqrt{4+\mu^{2}}$. This condition will enforce the quantization of the (not necessarily real) frequencies $\omega$, which will correspond to the (quasi)-normal modes as functions of the conformal weight $\Delta$ and of the parameters $T_{k}, \Omega_{k, 1}$ and $\Omega_{k, 2}$. The rather difficult problem is that the quantization condition cannot be expressed in terms of elementary functions of the parameters of the equations (2.20) and (2.28). We turn to this problem now.

\subsection{Isomonodromy and formal solutions}

Finding the quantization conditions from the problem above can be cast in terms of a reverse Riemann-Hilbert problem. Details can be found in [10, 11], and we will present here a summary of the ideas. Take a generic Fuchsian second order ordinary differential equation (ODE),

$$
y^{\prime \prime}(z)+p(z) y^{\prime}(z)+q(z) y(z)=0 .
$$

Frobenius method allows us to compute the solutions at a particular singular point $z_{k}$

$$
y_{k}^{+}(z)=\left(z-z_{k}\right)^{\alpha_{k}^{+}}\left(1+\mathcal{O}\left(z-z_{k}\right)\right), \quad y_{k}^{-}(z)=\left(z-z_{k}\right)^{\alpha_{k}^{-}}\left(1+\mathcal{O}\left(z-z_{k}\right)\right),
$$

where $\alpha_{k}^{ \pm}$are the solutions of the indicial equation at $z=z_{k}$. We will call $y_{k}^{ \pm}(z)$ constructed thusly the "Frobenius solutions" at $z_{k}$. These Frobenius solutions $y_{k}^{ \pm}(z)$ have the property that, under analytical continuation around $z_{k}$, yield

$$
y_{k}^{ \pm}\left(e^{2 \pi i}\left(z-z_{k}\right)+z_{k}\right)=e^{2 \pi i \alpha_{k}^{ \pm}} y_{k}^{ \pm}(z) .
$$

For our purposes, it will be more interesting to recast the Fuchsian ODE in terms of the matrix differential equation, called "Garnier system",

$$
\frac{d \Phi}{d z}=A(z) \Phi,
$$


where $\Phi(z)$ is a matrix of fundamental solutions

$$
\Phi(z)=\left(\begin{array}{cc}
y^{(1)}(z) & y^{(2)}(z) \\
w^{(1)}(z) & w^{(2)}(z)
\end{array}\right),
$$

where $y^{(1,2)}$ are any two linearly independent solutions of the ODE - they can be our Frobenius solutions $y_{k}^{ \pm}(z)$ as above, but we will treat with the generic case - and $w^{(1,2)}$ are auxiliary functions which depend explictly on the choice for the matrix $A(z)$

$$
w^{(1)}(z)=\frac{1}{a_{12}(z)}\left(\frac{d y^{(1)}}{d z}-a_{11}(z) y^{(1)}\right),
$$

with analogous expressions for $w^{(2)}$. It is straightforward to see that, if the solutions $y^{(1)}(z)$ and $y^{(2)}(z)$ are linearly independent, then $\Phi(z)$ is invertible. The matrix

$$
A(z)=\frac{d \Phi}{d z} \Phi(z)^{-1}
$$

can now then be thought of as a flat holomorphic connection, part of a two-dimensional gauge potential. The gauge symmetry is related to conjugation of the fundamental matrix $\Phi(z)$ by matrices with rational functions for entries. The gauge invariant quantities associated to $A(z)$ are the Wilson loop observables

$$
W\left[\gamma_{k}\right]=\mathcal{P} \exp \left[\oint_{\gamma_{k}} A(z) d z\right]=M_{\gamma_{k}},
$$

where $\gamma_{k}$ is a closed loop around the critical point $z_{k}$. From the ODE perspective, the matrix $M_{\gamma_{k}}$ implements the monodromy around $z_{k}$

$$
\Phi\left(e^{2 \pi i}\left(z-z_{k}\right)+z_{k}\right)=\Phi(z) M_{\gamma_{k}} .
$$

Because the choice of basis is gauge-dependent, the matrices $M_{\gamma_{k}}$ are defined up to conjugation. Their traces are readily given in terms of the indicial exponents $\alpha_{k}^{ \pm}$

$$
\operatorname{Tr} M_{\gamma_{k}}=2 e^{\pi i\left(\alpha_{k}^{+}+\alpha_{k}^{-}\right)} \cos \pi\left(\alpha_{k}^{+}-\alpha_{k}^{-}\right) .
$$

The sum of the indicial exponents $\alpha_{k}^{+}+\alpha_{k}^{-}$can be thought of as an abelian "charge": their values do not matter for the determination of the entries of the monodromy matrix. From the ODE perspective, their value can be modified with a "s-homotopic" transformation, like (2.19) and (2.27). We will consider $\alpha_{k}^{+}+\alpha_{k}^{-}=0$ from now on, and define $\sigma_{k}=$ $\alpha_{k}^{+}-\alpha_{k}^{-}=2 \alpha_{k}^{+}$.

Another set of conjugation-invariant quantities are the composite monodromies

$$
p_{k l}=\operatorname{Tr} M_{k} M_{l}=2 \cos \pi \sigma_{k l},
$$

which will permit us to solve the problem posed at the end of the last subsection. Let us define the Frobenius solution $\Phi_{k}(z)$ the fundamental matrix such that the first row is given by a pair of Frobenius solutions constructed at $z=z_{k}$ :

$$
\Phi_{k}(z)=\left(\begin{array}{cc}
y_{k}^{+}(z) & y_{k}^{-}(z) \\
w_{k}^{+}(z) & w_{k}^{-}(z)
\end{array}\right),
$$


where $y_{k}^{ \pm}(z)$ are like (2.34) and the second row is related to the first by (2.38). It can then be verified that the monodromy matrix around $z_{k}$ is diagonal, given by entries in (2.35)

$$
\Phi_{k}\left(e^{2 \pi i}\left(z-z_{k}\right)+z_{k}\right)=\Phi_{k}(z) M_{k}, \quad M_{k}=\left(\begin{array}{cc}
e^{+\pi i \sigma_{k}} & 0 \\
0 & e^{-\pi i \sigma_{k}}
\end{array}\right) .
$$

This is valid for any particular singular point $z_{k}$ provided we pick the solution $\Phi_{k}(z)$ constructed by the corresponding Frobenius solutions $y_{k}^{ \pm}(z)$. The hard question to answer is how this matrix $\Phi_{k}(z)$ will behave when we take it around a different singular point $z_{l}$. In order to relate the problem with the construction of the preceeding paragraphs, let us introduce the connection coefficients between the two Frobenius solutions

$$
y_{k}^{+}(z)=a_{k l} y_{l}^{+}(z)+b_{k l} y_{l}^{-}(z), \quad y_{k}^{-}(z)=c_{k l} y_{l}^{+}(z)+d_{k l} y_{l}^{-}(z) .
$$

Writing these in terms of the fundamental matrix, $\Phi_{k}(z)=\Phi_{l}(z) E_{k l}$, where $E_{k l}$ is the connection matrix. Now, since the monodromy matrix around $z_{l}$ is diagonal in terms of $\Phi_{l}(z)$, we can perform a change of basis to find

$$
\Phi_{k}\left(e^{2 \pi i}\left(z-z_{l}\right)+z_{l}\right)=\Phi_{k}(z) M_{l}, \quad M_{l}=E_{k l}^{-1}\left(\begin{array}{cc}
e^{+\pi i \sigma_{l}} & 0 \\
0 & e^{-\pi i \sigma_{l}}
\end{array}\right) E_{k l},
$$

where $\alpha_{l}^{ \pm}= \pm \sigma_{l} / 2$ are the two solutions for the indicial equations at $z=z_{l}$. Unfortunately, the matrix $E_{k l}$ is in general a very complicated function of the parameters entering the differential equation we started with.

For both the problems posed in the last section, (2.31) and (2.32), we are looking for parameters of the equations - (2.20) and (2.28), respectively - such that there is a special solution where a given behavior at $z=z_{k}$ and $z=z_{l}$ is specified. This means that one or more connection coefficients - entries of the connection matrix $E_{k l}$ - vanish. We will consider it to be lower triangular then. Let us now choose a basis where the monodromy matrix at $z=z_{k}, M_{k}$ is diagonal. Then, by the analysis above, the monodromy matrix at $z=z_{l}, M_{l}$ will be the conjugation of a diagonal matrix by a lower triangular matrix, which will again be lower triangular. Then it is a direct calculation to verify that the parameters of the composite monodromy between $z_{k}$ and $z_{l}$ introduced above will satisfy the quantization condition

$$
\sigma_{k l}=\sigma_{k}+\sigma_{l}+2 n, \quad n \in \mathbb{Z}
$$

Another direct calculation shows the converse, if the composite monodromy parameter $\sigma_{k l}$ satisfies the equation above, then the monodromy matrices $M_{k}$ and $M_{l}$ commute, and hence can be put simultaneously into the lower triangular form, which in turn means the vanishing of the connection coefficient and that there are solutions with the desired prescribed behavior at both points $z_{k}$ and $z_{l}$.

Translating this to the boundary conditions for $S(u)$ and $R(z),(2.31)$ and (2.32), respectively we find that, if one can compute the composite monodromy parameter $\sigma_{k l}$ in 
terms of the quantities in each ODE (2.20) and (2.28), the normal modes frequencies would be implicitly given by

$$
\begin{aligned}
\sigma_{01}\left(m_{1}, m_{2}, \beta, \Delta, u_{0}, \lambda_{\ell}\right) & =m_{1}+m_{2}+2 \ell, & & \ell \in \mathbb{Z}, \\
\sigma_{+\infty}\left(\theta_{k}, \Delta, z_{0}, \omega_{n}, \lambda_{\ell}\right) & =\theta_{\infty}+\theta_{+}+2 n, & & n \in \mathbb{Z} .
\end{aligned}
$$

Some comments are in order:

- We are thinking of the $\sigma_{i j}$ as a function of the parameters in the ODE. In particular, for (2.20), the list of arguments of the function comprehends $m_{1}, m_{2}, \beta, q_{1}, q_{2}$ from (2.21) and the accessory parameters $u_{0}$ and $Q_{0}$ from (2.22). Likewise, for (2.28), these are $\theta_{k}$ and $\Delta$ from (2.29) as well as $z_{0}, K_{0}$ from (2.30).

- The first condition pertains to the angular equation, between the regular singular points $z=0$ and $z=1$. The second to the radial equation between the points $z=r_{+}^{2}$ and $z=\infty$. In the second one, extra care has to be taken to pick the positive (real part of the) frequency solution.

In short, the message of the arguments given so far is that the solution of the problem rests on our hability to calculate the composite monodromy parameter $\sigma_{i j}$. That is where the isomonodromy method helps.

The isomonodromy method consists in finding a gauge where $A(z)$ in $(2.39)$ can be written as a partial fraction expansion

$$
A(z)=\frac{A_{0}}{z}+\frac{A_{1}}{z-1}+\frac{A_{t}}{z-t} .
$$

It was demonstrated explicitly in [11] that one can always achieve such a gauge for which the two independent solutions from first row of the fundamental matrix (2.37) satisfy the Heun equation for generic accessory parameters $t, K_{0}$. Given the expression for $A(z)$, we define the $\tau$-function

$$
\frac{d}{d t} \log \tau(t, \vec{\sigma})=\frac{1}{t} \operatorname{Tr}\left(A_{0} A_{t}\right)+\frac{1}{t-1} \operatorname{Tr}\left(A_{1} A_{t}\right),
$$

which can be shown [36] to be a function of only the invariant monodromy data like the parameters $\vec{\sigma}=\left\{\sigma_{k}, \sigma_{k l}\right\}$. For the Heun differential equation (2.28) - (2.20) is entirely analogous - the associated function $\tau$ satisfies a version of the Painlevé VI differential equation, and the composite monodromy data is encoded in the asymptotics of the solution as $t=0,1, \infty$ [37]. This differential equation is just the flat-curvature condition on $A(z)$ stated in terms of each coefficient matrix $A_{i}$. These equations are known in the literature as the Schlesinger equations.

Now, the $\tau$ function for the Painlevé VI system was shown to correspond to exact $c=1$ conformal blocks, and expansions near $t=0,1$ and $\infty$ were given in $[12,38]$, following the proposal [13] and subsequent proof [14] of the AGT conjecture. Perhaps more tantalizingly, the same $\tau$-function appears in the semiclassical expansion for Liouville conformal blocks [39] — see also [40]. In both cases the role of the Painlevé $\tau$-function 
as the character of the Virasoro algebra over a Verma module is paramount. Given the $\tau$-function expansion, we can phrase the problem of finding the composite monodromy parameters implicitly by

$$
\begin{gathered}
\left.t(t-1) \frac{d}{d t} \log \tau(t, \vec{\sigma})\right|_{t=z_{0}}=z_{0} \theta_{0} \theta_{+}+\left(z_{0}-1\right) \theta_{-} \theta_{0}+z_{0}\left(z_{0}-1\right) K_{0} \\
\left.\frac{d}{d t}\left[t(t-1) \frac{d}{d t} \log \tau(t, \vec{\sigma})\right]\right|_{t=z_{0}}=\left(\theta_{-}+\theta_{+}+\kappa_{1}\right) \theta_{0}=\frac{\theta_{0}}{2}\left(\theta_{-}+\theta_{+}-\theta_{0}+\theta_{\infty}\right),
\end{gathered}
$$

which again comes from the differential equation satisfied by the $\tau$ function, or, in other words, from the flat curvature condition on $A(z)$ as we change $t$. The conditions (2.53) apply directly to the radial equation (2.28), with a similar set of conditions for the angular equation (2.20). This deep connection between massive (scalar) perturbations of KerrAdS and Conformal Field Theory methods is quite surprising and still under development. Exact expansions for the Painlevé VI $\tau$-function can be found in the references above and those in the beginning of the subsection. These expansions, through the conditions (2.53), achieve an implicit solution to the problem posed in the last section, which is to find the parameters $\lambda_{\ell}, \omega_{n}$, defined in (2.50), corresponding to normal modes. We hope to explore the space of solutions and its implications in future work.

\section{Semi-classical conformal blocks and the near-extremal case}

In order to explore the relation between the isomonodromy and conformal field theory, let us rewrite the radial Heun equation $(2.28)$ in the $\mathrm{SL}(2, \mathbb{C})$ form

$$
\frac{d^{2} \Psi}{d z^{2}}+T(z) \Psi=0, \quad \Psi(z)=z^{1 / 2}(z-1)^{1 / 2}\left(z-z_{0}\right)^{1 / 2} \Pi(z)
$$

with $T(z)$ given by

$$
\begin{aligned}
T(z) & =\frac{\delta_{-}}{z^{2}}+\frac{\delta_{+}}{(z-1)^{2}}+\frac{\delta_{0}}{\left(z-z_{0}\right)^{2}}+\frac{\delta_{\infty}-\delta_{-}-\delta_{+}-\delta_{0}}{z(z-1)}+\frac{z_{0}\left(z_{0}-1\right) C_{0}}{z(z-1)\left(z-z_{0}\right)}, \\
\delta_{k} & =\frac{1}{4}-\frac{\theta_{k}^{2}}{4}, \quad k=-,+, 0, \quad \delta_{\infty}=-\frac{3}{4}-\frac{\mu^{2}}{4}=\frac{1}{4}-\frac{(\Delta-2)^{2}}{4}, \\
4 z_{0}\left(z_{0}-1\right) C_{0} & =-\frac{\lambda+\mu^{2} r_{0}^{2}-\omega^{2}}{r_{+}^{2}-r_{-}^{2}}-\theta_{-}^{2}+\theta_{+}^{2}+\left(2 z_{0}-1\right)\left(\theta_{0}^{2}-2\right) .
\end{aligned}
$$

We chose the radial equation for the treatment because the concept of (semi)-classical conformal blocks of section 2 is particularly useful here. The form given by (3.1) is known in classical uniformization theory, where the ratio of two linearly independent solutions $y^{(1)}(z), y^{(2)}(z)$ form the conformal map $w(z)$ between the upper-half plane $z$ and a geodesic quadrangle in the Poincaré plane, with internal angles $\phi_{i}= \pm \pi \sqrt{1-4 \delta_{i}}= \pm \pi \theta_{i}$. Since our application has imaginary $\theta_{i}$ for $z=0,1$, the inner and outer horizons, the analogy doesn't work there, we will leave the discussion about these points to the next subsection. In terms of the map $\zeta(z)=y^{(1)}(z) / y^{(2)}(z)$, equation (3.1) is written as

$$
\{\zeta(z) ; z\} \equiv \frac{\partial_{z}^{3} \zeta}{\partial_{z} \zeta}-\frac{3}{2}\left(\frac{\partial_{z}^{2} \zeta}{\partial_{z} \zeta}\right)^{2}=2 T(z)
$$

where the left hand side is known as the Schwarzian derivative. 
The uniformization problem can also be cast in terms of classical field theory. Let us consider again the equation satisfied by any of the entries of first row of the fundamental matrix $\Phi,(2.37)$

$$
\partial_{z}^{2} y-\left(\operatorname{Tr} A+\partial_{z} \log a_{12}\right) \partial_{z} y+\left(\operatorname{det} A-\partial_{z} a_{11}+a_{11} \partial_{z} \log a_{12}\right) y=0 .
$$

We would again like to cast it as a flat-connection condition (2.39). Since now the connection is traceless — the Wronskian $\operatorname{det} \Phi$ is constant — then the absence of the first derivative term requires that $a_{12}$ does not have any zeros on the complex plane. So we will take it to be a constant $a_{12}=\mu$. Now, using a Gauss parametrization of $\Phi(z)$ with the fields $\chi_{R}, \chi_{L}, \phi_{c}$,

$$
\Phi(z)=e^{\chi_{L} \sigma^{-}} e^{-\frac{1}{2} \phi_{c} \sigma^{3}} e^{\chi_{R} \sigma^{+}}=\left(\begin{array}{cc}
1 & 0 \\
\chi_{L} & 1
\end{array}\right)\left(\begin{array}{cc}
e^{-\frac{1}{2} \phi_{c}} & 0 \\
0 & e^{\frac{1}{2} \phi_{c}}
\end{array}\right)\left(\begin{array}{cc}
1 & \chi_{R} \\
0 & 1
\end{array}\right),
$$

we define the holomorphic connection $\mathcal{A}_{z}=\left[\partial_{z} \Phi(z)\right] \Phi^{-1}(z)$ in the same way as in the preceding section and its complex conjugate as $\mathcal{A}_{z^{*}}=\left(\mathcal{A}_{z}\right)^{\dagger}$. It is a straightforward exercise to show that the constraint that the upper off-diagonal element is constant $\mathcal{A}_{12}=\overline{\mathcal{A}}_{21}=\mu$ - an "oper" - reduces the flat connection condition to the Liouville equation

$$
\partial_{z} \partial_{\bar{z}} \phi_{c}=\mu^{2} e^{\phi_{c}}
$$

encoding the fact that the original metric in $\zeta$ coordinates has constant negative curvature $-4 \mu^{2}$. The classical solution to the Liouville equation is given in terms of $\zeta(z)$

$$
\phi_{c}\left(z, z^{*}\right)=\log \left(-\frac{2}{\mu^{2}} \frac{\partial_{z} \zeta \partial_{z^{*}} \zeta^{*}}{\left(\zeta(z)-\zeta^{*}\left(z^{*}\right)\right)^{2}}\right) .
$$

The field $\phi_{c}\left(z, z^{*}\right)$ has obvious singularities when $\zeta=\zeta^{*}$, which is the image of the real line $z=z^{*}$. For $z$ on the real line such that $\zeta(z)$ is analytic, the image is composed of straight lines or circular arcs. However, $\zeta(z)$ has singular points on the real line, tied to the singular points of (3.1), where the image develops a wedge or cusp of angle $\pi \theta_{k}$ - we will see in the next subsection what happens for imaginary $\theta_{k}$. In either case, we can verify that, up to a translation and a rotation, the map $\zeta(z)$ near $z_{k}$ behaves as

$$
\zeta(z)=\left(z-z_{k}\right)^{\theta_{k}}(1+\ldots), \quad z \simeq z_{k},
$$

which translates to the asymptotic behavior of the Liouville field via the classical solution (3.9).

Quantum Liouville field theory is built upon the action

$$
\mathcal{S}_{L}=\frac{1}{8 \pi b^{2}} \int_{D} d^{2} \xi\left[\partial_{a} \phi_{c} \partial^{a} \phi_{c}+2 \mu^{2} e^{\phi_{c}}\right]
$$

see [41] for a review. The constant $b^{2}$ has the interpretation of Planck's constant and the semiclassical limit will then be defined by $b \rightarrow 0$. The domain of integration $D$ will be taken to be the sphere with flat metric $\eta_{a b}=\delta_{a b}$. In the usual parametrization of the kinetic 
term, we define the Liouville field $\phi=\phi_{c} / 2 b$. The theory can be seen to be conformally invariant at the quantum level if the field transforms as

$$
\phi^{\prime}\left(z^{\prime},\left(z^{\prime}\right)^{*}\right)=\phi\left(z, z^{*}\right)-\frac{Q}{2} \log \left|\frac{\partial z^{\prime}}{\partial z}\right|^{2},
$$

where $Q=b+1 / b$. The Hilbert space of Liouville field theory corresponds to highest weight states constructed from the action of vertex operators $V_{\Delta(\alpha)}=: e^{2 \alpha \phi}$ : on a $\operatorname{SL}(2, \mathbb{C})$ invariant "vacuum" state $|0\rangle$. The parameter $\Delta$ is the conformal dimension of the operator and its dependence on $\alpha$ will be given below. Each of the $V_{\Delta(\alpha)}$ generates an infinite tower of states by action of the Virasoro algebra $\left\{L_{n}\right\}$, where

$$
T(z)=-(\partial \phi)^{2}+Q \partial^{2} \phi=\sum_{n=-\infty}^{\infty} L_{n} z^{-n-2},
$$

satisfying $\left[L_{n}, L_{m}\right]=(n-m) L_{n+m}+\frac{c}{12} \delta_{n+m, 0}$, with $c=1+6 Q^{2}$. Here $T(z)=-2 \pi T_{z z}(z)$, the appropriate component of the stress-energy tensor of the Liouville field, whose conservation law implies that it is holomorphic. There are analogous formulas for the antiholomorphic sector, which we will omit. With some effort [42], one can show that $V_{\Delta(\alpha)}$ is a primary operator,

$$
e^{2 \alpha \phi^{\prime}\left(z^{\prime},\left(z^{\prime}\right)^{*}\right)}=\left(\frac{\partial z^{\prime}}{\partial z}\right)^{-\Delta(\alpha)}\left(\frac{\partial\left(z^{\prime}\right)^{*}}{\partial z^{*}}\right)^{-\bar{\Delta}(\alpha)} e^{2 \alpha \phi\left(z, z^{*}\right)},
$$

where $\Delta(\alpha)=\alpha(Q-\alpha)=\bar{\Delta}(\alpha)$. Equivalently, one can phrase the transformation law in terms of the OPE

$$
T(z) V_{\Delta}\left(z_{0}, z_{0}^{*}\right) \simeq \frac{\Delta(\alpha)}{\left(z-z_{0}\right)^{2}} V_{\Delta}\left(z_{0}, z_{0}^{*}\right)+\frac{1}{z-z_{0}} \partial_{z_{0}} V_{\Delta}\left(z_{0}, z_{0}^{*}\right) .
$$

In the quantum theory, $e^{-\frac{1}{2} \phi_{c}} \equiv V_{\Delta(-b / 2)}$ corresponds to a degenerate Virasoro module at level 2 , because the operator

$$
\left(L_{-1}^{2}+b^{2} L_{-2}\right) e^{-\frac{1}{2} \phi_{c}}
$$

has a null norm and therefore must decouple from local operators in correlation functions. The corresponding Ward identity satisfied by $e^{-\frac{1}{2} \phi_{c}}$ is

$$
\left\langle\left(L_{-1}^{2}+b^{2} L_{-2}\right) e^{-\frac{1}{2} \phi_{c}}(z) X\left(\left\{z_{i}\right\}\right)\right\rangle=0,
$$

where $X\left(\left\{z_{i}\right\}\right)$ is a generic local operator. We will consider the case where $X\left(\left\{z_{i}\right\}\right)$ is a product of vertex operators $V_{\Delta_{i}}$, and each corresponding $\alpha_{i}$ of order $1 / b$ so it can be thought of as a "heavy operator", setting a background over which $e^{-\frac{1}{2} \phi_{c}}$ reacts. Using the OPE between $T(z)$ and the primary operator $e^{-\frac{1}{2} \phi_{c}}$ from (3.15) one can see that

$$
L_{-1} e^{-\frac{1}{2} \phi_{c}}(z)=\partial_{z} e^{-\frac{1}{2} \phi_{c}}(z), \quad L_{-2} e^{-\frac{1}{2} \phi_{c}}(z)=: T(z) e^{-\frac{1}{2} \phi_{c}}(z): .
$$


Therefore, by considering the subcase where $X$ is the product of four primary operators, projected to an intermediate channel of conformal dimension $\Delta$ via a projection operator $\Pi(\Delta)$ (normal ordering of each operator is implicit),

$$
X\left(z_{a}, z_{b}, z_{c}, z_{d}\right)=e^{2 \alpha_{a} \phi\left(z_{a}\right)} e^{2 \alpha_{b} \phi\left(z_{b}\right)} \Pi(\Delta) e^{2 \alpha_{c} \phi\left(z_{c}\right)} e^{2 \alpha_{d} \phi\left(z_{d}\right)},
$$

and setting $z_{a}=\infty, z_{b}=z_{0}, z_{c}=1$ and $z_{d}=0$, the Ward identity turns to (3.1) in the semiclassical limit $b \rightarrow 0$. This is a direct consequence of the OPE between $T(z)$ and the primary operators (3.15), whose singular terms will yield (3.4) and the wavefunction is identified with $\Psi(z)=\left\langle e^{-\frac{1}{2} \phi_{c}} X\right\rangle$. The accessory parameter $C_{0}$ is given by the logarithm derivative of the correlation function of $X$

$$
\begin{aligned}
C_{0} & =b^{2} \frac{d}{d z_{0}} \log F\left(z_{0}\right), \\
F\left(z_{0}\right) & =\lim _{z_{a} \rightarrow \infty} z_{a}^{-2 \Delta_{a}}\left\langle V_{\Delta_{a}}\left(z_{a}\right) V_{\Delta_{b}}\left(z_{0}\right) \Pi(\Delta) V_{\Delta_{c}}(1) V_{\Delta_{d}}(0)\right\rangle \simeq e^{-S_{\mathrm{cl}}\left(\Delta_{i}, \Delta\right)},
\end{aligned}
$$

where at the last inequality we took the semiclassical approximation for the 4-point function as the value of the classical action of the Liouville field subjected to the boundary conditions at the singular points (3.10). For the problem of our interest, the role of the projection operator singles out one intermediate conformal channel with definite conformal dimension $\Delta$, which we will take to be "heavy": $\Delta=\mathcal{O}\left(b^{-2}\right)$, as in (3.24) below. See figure 1 for a semiclassical picture. The function $F\left(z_{0}\right)$ is the semiclassical conformal block, and could in principle be obtained from the representation theory of Virasoro algebra. An expansion for small $z_{0}$ can be obtained from [39]. In terms of the radial equation, a large, and negative, value for $z_{0}$ is accomplished near the extremal limit $r_{+} \rightarrow r_{-}$:

$$
z_{0}=\frac{r_{0}^{2}-r_{-}^{2}}{r_{+}^{2}-r_{-}^{2}}=-\frac{r_{+}^{2}+2 r_{-}^{2}+a^{2}+b^{2}+1}{r_{+}^{2}-r_{-}^{2}}
$$

By the same token, a large intermediate dimension means a large intermediate monodromy parameter, which in turn can be heuristically assigned to a finite frequency in (3.4). To sum up, the semi-classical approximation is then expected to describe the radial equation in the near-extremal, finite frequency limit.

As an important comment, in the semiclassical limit considered below, the relation between the accessory parameter $C_{0}$ and the conformal dimension of the intermediate channel is a bijection, as expressed in relations obtained in the preceding section like (2.50). From this one deduces that the relevant quantity for finding the normal modes of (3.1) is not exactly the generic four-point function but the conformal block. The integer factors arising in the expansion correspond to the Virasoro descendants of the intermediate channel.

\subsection{Entropy and Liouville momenta}

The classical asymptotics of the Liouville field (3.10) means that the "physical" metric

$$
d s^{2}=e^{\phi_{c}} d z d z^{*}
$$


will develop a cusp-like singularity at $z=z_{k}$ for real $\theta_{k}$. This is indeed the case for the singularities at $z=\infty$ and $z=z_{0}$ in the radial equation (2.28). The latter is real due to the fact that $r_{0}$ is purely imaginary and therefore so is the "temperature" $T_{0}$. For the angular equation (2.20), all the singularities have real indicial exponents, so the semiclassical interpretation of the corresponding four-point function as living on a sphere is still valid. As discussed above, the geometric interpretation in these cases is of a deficit angle $\pi \theta_{k}$ at $w_{k}=w\left(z_{k}\right)$, and the singular points are related to local operators $: e^{2 \alpha_{k} \phi\left(z_{k}\right)}$ : with scaling dimension

$$
\delta_{k}=b^{2} \Delta_{k}=\eta_{k}\left(1+b^{2}-\eta_{k}\right) \simeq \eta_{k}\left(1-\eta_{k}\right),
$$

where $\eta_{k}=b \alpha_{k}$. For $\eta_{k}$ real and smaller than $1 / 2$, the geometry of the physical metric develops a cusp at $z_{k}$. These will be associated with the singular points at $z=\infty$ and $z=z_{0}$. From the relation between the Liouville field and the map $\zeta(z)$, we learn that the deficit angle $\pi \theta_{k}=2 \eta_{k}$, and hence vertex operators with $\alpha_{k}>1 / 2 b \simeq Q / 2$ do not have a semiclassical geometrical interpretation. This is called the Seiberg bound. At the quantum mechanical level, we learn — see, for instance, [41] — that

$$
V_{\Delta(\alpha)}=C(\alpha) V_{\Delta(Q-\alpha)},
$$

where $C(\alpha)$ is called the "reflection amplitude" and is a known function of $\alpha$.

For the radial equation (2.28), the singularities at $z=z_{0}$ and $z=\infty$ have real $\delta_{k}$ associated to them. The picture above is well suited provided we use the reflection property (3.25) to assign the singularity to an operator - possibly heavy - with $\alpha \approx Q$. For instance, the insertion at $z=\infty$ can be seen to correspond to a "heavy operator" $[18,19]$ $\alpha_{\infty}=\eta_{\infty} / b$ with

$$
\eta_{\infty}=\frac{\Delta-1}{2}, \frac{3-\Delta}{2}
$$

with the first solution being proper for $\Delta<2$ and the second for $\Delta>2$, see [41] for a detailed discussion.

On the other hand, the singularities at $z=0,1$, corresponding to the inner and outer horizon have the property that

$$
\theta_{ \pm}=\frac{i}{2 \pi}\left(\frac{\omega-m_{1} \Omega_{ \pm, 1}-m_{2} \Omega_{ \pm, 2}}{T_{ \pm}}\right)=\frac{i}{2 \pi} \delta S_{ \pm}
$$

where $\delta S_{ \pm}$is the variation of entropy of the black hole horizon at $r=r_{ \pm}$, respectively, due to the admittance of energy $\omega$ and angular momenta $m_{1}$ and $m_{2} . \delta S_{ \pm}$is then real and finite for physically reasonable parameters black hole parameters $M, a_{1}$ and $a_{2}$.

In this case the "physical" metric near $z_{ \pm}=0,1$ is approximately given by

$$
d s^{2}=e^{\phi_{c}} d z d z^{*}=\frac{1}{2 \mu^{2}} \frac{d z^{\prime} d\left(z^{\prime}\right)^{*}}{\sin ^{2}\left(\frac{\delta S_{ \pm}}{2 \pi} \frac{z^{\prime}+\left(z^{\prime}\right)^{*}}{2}\right)}
$$

where we made the transformation $z-z_{ \pm}=e^{z^{\prime}}$ in the second step $\left(z_{ \pm}=0,1\right)$. The approximation is valid for $z^{\prime}$ sufficiently close to zero. Because of the identification of the 


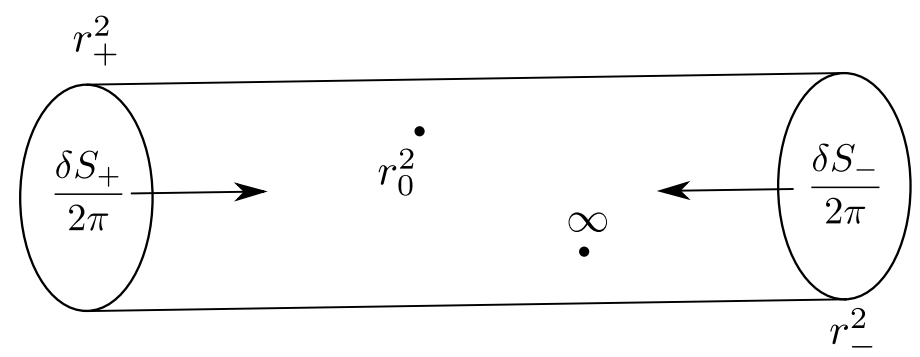

Figure 2. The "world-sheet" Liouville description of the conformal block related to the black-hole scattering. While the insertions of the angular equation (2.20) are all cusp-like singularities, the radial equation (2.28) possesses conformal boundaries related to the inner and outer horizon. The effective geometry is that of a cylinder, and the entropy increase of the horizons is equal to the Liouville momentum inserted at each horizon.

imaginary part of $z^{\prime}$, the metric is actually defined on a cylinder. At the quantum level, the vertex operator at $z_{ \pm}=0,1$ corresponds to $V_{\Delta\left(\alpha_{ \pm}\right)}\left(z_{ \pm}\right)$, with

$$
\alpha_{ \pm}=\frac{Q}{2}+i \frac{\delta S_{ \pm}}{4 \pi b}
$$

and, in our conventions, we consider $z$ to be the complex plane. If we map the plane to the cylinder by $w=\log z$, the Liouville momentum - the zero mode for $\Pi=\dot{\phi}-$ will be shifted because of the anomalous transformation law (3.12)

$$
\partial_{z} \phi \rightarrow \frac{1}{z}\left(\partial_{w} \phi-Q / 2\right)
$$

and then $\delta S_{ \pm} / 2 \pi$ is the Liouville momentum injected at $z_{ \pm}$. We illustrate the Liouville diagram computed by the conformal block in figure 2 .

The geometrical picture actually helps us writing an approximate expansion for the composite monodromy in the near horizon limit $r_{+} \rightarrow r_{-}$. This limit is characterized not only by a large - and negative value of $z_{0}$, but also large values for $\theta_{+}$and $\theta_{-}$, which scale as $z_{0}$. Although there are no "light" operators, there is a hierarchy because $\theta_{0}$ and $\delta_{\infty}$ do not scale with $z_{0}$. We can combine a method given by [43] and notions of thermality to write an approximate expression for the accessory parameter $C_{0}$ in terms of the composite monodromy parameter between $z=1$ and $z=\infty$, which we will call $\delta_{1, \infty}=\delta$. The limit is complicated by the generic scaling behavior:

$$
\delta_{ \pm}=\mathcal{O}\left(z_{0}^{2}\right), \quad \delta_{+}-\delta_{-}=\mathcal{O}\left(z_{0}\right), \quad \delta_{0}, \Delta=\mathcal{O}\left(z_{0}^{0}\right), \quad C_{0}=\mathcal{O}\left(z_{0}^{-1}\right) .
$$

By transforming $u=1 / z$, we bring the singular points of interest to 0 and 1 . The equation (3.1) is

with

$$
\frac{d^{2} \bar{\Psi}}{d u^{2}}+\bar{T}(u) \bar{\Psi}=0, \quad \bar{\Psi}(u)=u \Psi(z=1 / u)
$$

$$
\begin{aligned}
\bar{T}(u) & =\frac{\delta_{\infty}}{u^{2}}+\frac{\delta_{+}}{(u-1)^{2}}+\frac{\delta_{0}}{\left(u-u_{0}\right)^{2}}+\frac{\delta_{-}-\delta_{\infty}-\delta_{+}-\delta_{0}}{u(u-1)}+\frac{u_{0}\left(u_{0}-1\right) \tilde{C}_{0}}{u(u-1)\left(u-u_{0}\right)}, \\
u_{0} & =\frac{1}{z_{0}}, \quad \tilde{C}_{0}=-z_{0}^{2} C_{0}-2 z_{0} \delta_{0} .
\end{aligned}
$$


Now we observe that the solution $\bar{\Psi}(u)$ of (3.32) corresponds to the classical profile of the Liouville field $e^{-\frac{1}{2} \phi_{c}}$ in the presence of the "heavy operators" $V_{\Delta_{i}}$. Upon a conformal transformation $\tilde{u}(u)$, it is straightforward to verify that $\tilde{\Psi}(\tilde{u})=\left(\partial_{u} \tilde{u}\right)^{1 / 2} \bar{\Psi}(u)$ satisfies:

$$
\partial_{\tilde{u}} \tilde{\Psi}+\tilde{T}(\tilde{u}) \tilde{\Psi}=0
$$

where

$$
\tilde{T}(\tilde{u})=\left(\partial_{u} \tilde{u}\right)^{-2}\left(\bar{T}(u)-\frac{1}{2}\{\tilde{u} ; u\}\right) .
$$

By choosing $\tilde{u}$ such that:

$$
\frac{1}{2}\{\tilde{u} ; u\}=\frac{\delta_{\infty}}{u^{2}}+\frac{\delta_{+}}{(u-1)^{2}}+\frac{\delta_{-}-\delta_{\infty}-\delta_{+}-\delta_{0}}{u(u-1)}
$$

one "reduces" $\tilde{T}(\tilde{u})$ to two terms:

$$
\tilde{T}(\tilde{u})=\left(\partial_{u} \tilde{u}\right)^{-2}\left(\frac{\delta_{0}}{\left(u-u_{0}\right)^{2}}+\frac{u_{0}\left(u_{0}-1\right) \tilde{C}_{0}}{u(u-1)\left(u-u_{0}\right)}\right),
$$

and, since the second term is large in the large $z_{0}$ limit, we can give an approximate solution for $\tilde{\Psi}$ using a WKB approximation:

$$
\begin{aligned}
\tilde{\Psi}(\tilde{u}) & =\tilde{\Psi}_{0} \exp \left[ \pm \int_{\tilde{u}_{i}}^{\tilde{u}} d \tilde{u}^{\prime}\left(\partial_{u} \tilde{u}^{\prime}\right)^{-1} \sqrt{\frac{\delta_{0}}{\left(u\left(\tilde{u}^{\prime}\right)-u_{0}\right)^{2}}+\frac{u_{0}\left(u_{0}-1\right) \tilde{C}_{0}}{u\left(\tilde{u}^{\prime}\right)\left(u\left(\tilde{u}^{\prime}\right)-1\right)\left(u\left(\tilde{u}^{\prime}\right)-u_{0}\right)}}\right] \\
& =\tilde{\Psi}_{0} \exp \left[ \pm \sqrt{\delta_{0}} \int_{u_{i}}^{u} \frac{d u^{\prime}}{u-u_{0}} \sqrt{\frac{\left(u^{\prime}-u_{+}\right)\left(u^{\prime}-u_{-}\right)}{u^{\prime}\left(u^{\prime}-1\right)}}\right]
\end{aligned}
$$

where we assumed $\partial_{u} \tilde{u} \neq 0$ in the path of integration. The constants $u_{ \pm}$are given by:

$$
u_{ \pm}=-\frac{D+1-u_{0}(D+2)}{2}\left[1 \pm \sqrt{1+\frac{4 u_{0}\left(1-u_{0}\right)(D+2)}{\left(D+1-u_{0}(D+2)\right)^{2}}}\right], \quad D=\frac{C_{0}}{u_{0} \delta_{0}} .
$$

One notes that $D$ is of order $\mathcal{O}\left(z_{0}^{0}\right)$. For small $u_{0}$, one has:

$$
u_{+}=-\left(D_{0}+1\right)+\mathcal{O}\left(u_{0}\right), \quad u_{-}=\frac{D_{0}+2}{D_{0}+1} u_{0}+\mathcal{O}\left(u_{0}^{2}\right)
$$

where $D_{0}=\lim _{u_{0} \rightarrow 0} C_{0} / u_{0} \delta_{0}$. Both $u_{ \pm}$are negative for large enough frequency.

Now, equation (3.37) can be solved explicitly in terms of hypergeometric functions:

$$
\tilde{u}=\frac{u^{\frac{1}{2}\left(\theta_{+}-h\right)}(u-1)^{\frac{1}{2}\left(1-\theta_{+}\right)}{ }_{2} F_{1}\left(\frac{1}{2}\left(1+\theta_{\infty}-\theta_{+}+h\right), \frac{1}{2}\left(1-\theta_{\infty}-\theta_{+}+h\right) ; 1-h ; u^{-1}\right)}{u^{\frac{1}{2}\left(\theta_{+}+h\right)}(u-1)^{\frac{1}{2}\left(1-\theta_{+}\right)} 2 F_{1}\left(\frac{1}{2}\left(1+\theta_{\infty}-\theta_{+}-h\right), \frac{1}{2}\left(1-\theta_{\infty}-\theta_{+}-h\right) ; 1+h ; u^{-1}\right)}
$$

where $h^{2}=1+\theta_{-}^{2}-\theta_{0}^{2}$ and $\theta_{\infty}=\Delta-2$. The function $\tilde{u}$ is defined up to a Möbius map, stemming from the choice of hypergeometric functions in the numerator and denominator. 
The choice above guarantees that we have positive Liouville momentum entering $u=u_{0}$ and that we have a diagonal monodromy near $u=\infty$. The function $\tilde{u}$ contributes the term:

$$
\begin{aligned}
\left(\partial_{u} \tilde{u}\right)^{-1 / 2}= & W^{-1 / 2} u^{\frac{1}{2}\left(\theta_{+}+h\right)}(u-1)^{\frac{1}{2}\left(1-\theta_{+}\right)} \\
& \times{ }_{2} F_{1}\left(\frac{1}{2}\left(1+\theta_{\infty}-\theta_{+}-h\right), \frac{1}{2}\left(1-\theta_{\infty}-\theta_{+}-h\right) ; 1-h ; u^{-1}\right),
\end{aligned}
$$

to the solution $\bar{\Psi}(u)$, where $W$ is a constant.

From the splitting $\bar{\Psi}(u)=\left(\partial_{u} \tilde{u}\right)^{-1 / 2} \tilde{\Psi}(\tilde{u}(u))$ we have an approximate solution for (3.1). Calculating the monodromy between the points $u=1$ and $u=0$, corresponding respectively to the outer horizon and infinity is then a straightforward exercise in complex analysis [43]. The integral in (3.40) is related to incomplete elliptic integrals, which for the contour $C_{01}$ around $u=0$ and $u=1$ picks a complete elliptic integral of the third kind $\Pi(\nu, k)$ :

$$
\begin{aligned}
\oint_{C_{01}} \frac{d u}{u-u_{0}} \sqrt{\frac{\left(u-u_{+}\right)\left(u-u_{-}\right)}{u(1-u)}=} & -\frac{4 u_{-}\left(u_{+}-u_{0}\right)}{u_{0} \sqrt{u_{+}\left(u_{-}-1\right)}} \Pi\left(\frac{u_{-}-u_{0}}{u_{0}\left(u_{-}-1\right)}, \sqrt{\frac{u_{+}-u_{-}}{u_{+}\left(1-u_{-}\right)}}\right) \\
& -\frac{4 u_{-}}{\sqrt{u_{+}\left(u_{-}-1\right)}} \Pi\left(\frac{1}{1-u_{-}}, \sqrt{\frac{u_{+}-u_{-}}{u_{+}}}\right) .
\end{aligned}
$$

For small $u_{0}$, we use the expansion:

$$
\Pi\left(\nu, k^{2}\right)=\frac{1}{1-\nu}\left[\left(1+\mathcal{O}\left(k^{2}\right)\right) \log \frac{4}{k^{\prime 2}}-\sqrt{\nu} \log \frac{1+\sqrt{\nu}}{\sqrt{|1-\nu|}}\right]
$$

with $k^{\prime 2}=1-k^{2}$ and $0<\nu<1$, and we have the asymptotic behavior:

$$
\oint_{C_{01}} \frac{d u}{u-u_{0}} \sqrt{\frac{\left(u-u_{+}\right)\left(u-u_{-}\right)}{u(1-u)}}=-2 \frac{D_{0}+2}{D_{0}} \sqrt{\left|D_{0}+1\right|} \log \left(-u_{0}\right)+\mathcal{O}\left(u_{0}, u_{0} \log \left(-u_{0}\right)\right)
$$

At this point one has to check whether the WKB approximation is valid on the contour $C_{01}$. The conditions for the validity were considered by [44], where it is shown that the precision of the WKB series is tied to the existence of a zero or singularity of the integrand at some $\bar{u}$ where the divergence is milder than $|u-\bar{u}|^{-1+\epsilon}$, with $\epsilon>0$. In our application this is not the case near $u_{0}$, but it is near $u_{+}, u_{-}, 0,1$. For the contour $C_{01}$, the deformation should be valid for intermediate ranges of $u_{0}$ or in those cases where $u_{-}>u_{0}-$ which requires $D_{0}<-1$. In any case, the contour can be deformed in the complex plane to avoid the pole at $u_{0}$ and errors can be estimated to be of order $u_{0} \log \left(-u_{0}\right)$.

Finally, one notes that, while the solutions $\bar{\Psi}(u)$ in (3.32) is analytic everywhere except at the critical points $u=0, u_{0}, 1, \infty$, the approximation $\tilde{\Psi}(\tilde{u}(u))$ has extra branching points at $u=u_{ \pm}$. Thus, the approximation used here displays the Stokes phenomenon: each of the solutions at (3.40) can only approximate the exact solution on regions radiating from $u_{ \pm}$. Since $u_{ \pm}$is a simple zero in the radical of the integrand, the situation here is completely analogous to the Stokes phenomenon arising from the WKB approximation of the Airy function near a classical turning point: the principal region is limited to the anti-Stokes 
line $\left|\arg \left(u-u_{ \pm}\right)\right|<\pi / 3$. It can be seen that the contour encompassing $u=0,1$ considered can be seen to keep to the principal region, since we can deform $C_{01}$ to cross the real line in between $u_{-}$and 0 . In the classical mechanics analogy, we are never going over the classical turning point by following through $C_{01}$. Therefore each of the two solutions (3.40) are good throughout the contour, and no extra Stokes parameters are picked.

With these provisions, the contribution for the monodromy from the $\left(\partial_{u} \tilde{u}\right)^{-1 / 2}$ term is readily computed. Since it is given by a hypergeometric function, we know that a curve encompassing $u=0$ and $u=1$ can be deformed to a curve going around $u=\infty$, and the monodromy phase then equals $i \pi(1 \pm h)$ from the exponent of the $u$ term in (3.44).

The CFT interpretation of this composite monodromy stems from the OPE between the "light" operator $e^{\frac{1}{2} \phi_{c}}=V_{\Delta(-b / 2)}$ and the intermediate conformal channel obtained from the fusion between two of the operators in (3.21):

$$
V_{\Delta_{a}}\left(u_{a}\right) V_{\Delta_{b}}\left(u_{b}\right)=\left(u_{a}-u_{b}\right)^{\Delta-\Delta_{a}-\Delta_{b}}\left(V_{\Delta}\left(u_{a}\right)+\mathcal{O}\left(\left(u_{a}-u_{b}\right)\right)\right) .
$$

Now, since the light operator $V_{\Delta(-b / 2)}$ is associated to a degenerate representation - it has a null descendant of level $2(3.16)$ - then the OPE between it and $V_{\Delta}$ has only two channels: $\Delta_{ \pm}=\alpha_{ \pm}\left(Q-\alpha_{ \pm}\right)$, where $\alpha_{ \pm}=\alpha \pm b / 2$. Near $u_{a}$ we have again:

$$
V_{\Delta}\left(u_{a}\right) V_{\Delta(-b / 2)}(u)=\left(u-u_{a}\right)^{\Delta_{ \pm}-\Delta-\Delta(b / 2)} V_{\Delta_{ \pm}}+\ldots
$$

and, assuming that $V_{\Delta}\left(u_{a}\right)$ is heavy, we define $\delta=b^{2} \Delta$ and in the semiclassical limit the behavior near $u_{a}$ is approximately given by $u^{\frac{1}{2}(1 \pm \sqrt{1-4 \delta})}$. Defining $\sigma$ as $4 \delta=1-\sigma^{2}$, we can write the monodromy picked around $u_{a} \rightarrow 0$ as the phase $i \pi(1 \pm \sigma)$.

Equating both results, we arrive at the result for the composite monodromy parameter $\sigma$ for large negative $z_{0}$ and $D_{0}<-1$ as defined above

$$
\sigma=h+\frac{2}{i \pi} \frac{z_{0} C_{0}+2 \delta_{0}}{z_{0} C_{0}} \sqrt{\left|z_{0} C_{0}+\delta_{0}\right|} \log \left(-z_{0}\right)+\mathcal{O}\left(\left(-z_{0}\right)^{-1},\left(-z_{0}\right)^{-1} \log \left(-z_{0}\right)\right),
$$

where the signs were chosen to recover the right limit as $C_{0}$ vanishes. For large $C_{0}$, the $\delta_{0}$ factor can be disregarded. As we can see from (3.4), this result is consistent with the fact that the intermediate channel dimension is large for large $z_{0}$. This result can be used to determine the spectrum of quasi-normal modes in the limit where $\delta S_{ \pm}$is large. We will postpone this study to future work.

\section{Discussion}

In this paper we reviewed the relation between four dimensional classical conformal blocks and the propagation of fields in AdS spaces. We also estabilished the relation of the scattering problem of a scalar field in a Kerr-AdS background in five dimensions to two dimensional conformal blocks, and argued that the near-extremal limit of the black hole is described by semiclassical Liouville conformal blocks, in a picture somewhat different from [45]. We also saw that singular points of the radial equation are physically tied to the positions of the horizons, the point at infinity and the negative root of $\Delta_{r}(2.12)$. While 
the points are related to usual vertex operators in Liouville field theory, we argued that the singular points at the inner and outer horizon saturate the Seiberg bound, with the Liouville momentum of the operator given the variation of the entropy of the black hole horizon by absorption of the incoming wave. This gives a two-dimensional geometrical description of the process, as the Liouville correlator on the cylinder with a number of marked points - see figure 2. Remarkably, the corresponding Liouville description seems unitary in itself.

It would be very interesting to see whether this classical correspondence also holds at the quantum level. Features like the central charge have been shown several times to be related to the black hole entropy, so it is tempting to see whether one can push the analogy further and recover information from the quantum theory from the description provided here. Also, the intringuing way two-dimensional CFTs keep appearing in higher dimensional problems may herald some deeper connection to quantum gravity. Interestingly, the same connection between CFT and integrability is also frequent, common and mysterious. Mathematically, it is a bit of a surprise that the full Virasoro algebra - with $c=1-$ seems relevant to the connection problem of the four-singularity Fuchsian equation. All of these points deserve better understanding.

Lastly, the analytical tools developed here, specially the inspection of (2.50), may help understand the constraints posed by holography to the RG flow in a generic setting. As important properties of non-abelian gauge theories, such as confinement and the conformal window, depend crucially on the specific way the theory flows from the UV to the IR, it is important to understand in detail which flows can be holographically described. Implementation of more realistic elements such that spinorial fields and flavor should be still mathematically tractable by the tools developed here. Mathematically the spinor equation on a Kerr-AdS background has an entirely analogous treatment in terms of Heun's equations as described. Also, with controllable parameters even at $T=0$ - the extremal case, one can study the zero temperature limit. Since in the zero temperature a conformal group is still present [32], there should be still a massless sector there. The transition may further help shed light on the physical properties listed above from a holographic perspective, taking into account recent progress on QCD and SUSY QCD made in [46, 47].

\section{Acknowledgments}

The authors are greatly thankful to M. Guica for discussions, ideas and suggestions, and for the referee for spotting an error in the previous calculation for the composite monodromy in the near extremal limit. We would also like to thank F. Novaes, A. Queiroz, F. Rudrigues, D. Crowdy, G. Vasconcelos. BCdC is thankful to PROPESQ/UFPE and FACEPE for support under grant no. APQ-0051-1.05/15.

Open Access. This article is distributed under the terms of the Creative Commons Attribution License (CC-BY 4.0), which permits any use, distribution and reproduction in any medium, provided the original author(s) and source are credited. 


\section{References}

[1] D. Marolf, Black holes, AdS and CFTs, Gen. Rel. Grav. 41 (2009) 903 [arXiv:0810.4886] [INSPIRE].

[2] L. Girardello, M. Petrini, M. Porrati and A. Zaffaroni, Novel local CFT and exact results on perturbations of $N=4$ super Yang-Mills from AdS dynamics, JHEP 12 (1998) 022 [hep-th/9810126] [INSPIRE].

[3] O. DeWolfe, D.Z. Freedman, S.S. Gubser and A. Karch, Modeling the fifth-dimension with scalars and gravity, Phys. Rev. D 62 (2000) 046008 [hep-th/9909134] [INSPIRE].

[4] E. Berti, V. Cardoso and A.O. Starinets, Quasinormal modes of black holes and black branes, Class. Quant. Grav. 26 (2009) 163001 [arXiv:0905.2975] [INSPIRE].

[5] G.T. Horowitz and V.E. Hubeny, Quasinormal modes of AdS black holes and the approach to thermal equilibrium, Phys. Rev. D 62 (2000) 024027 [hep-th/9909056] [INSPIRE].

[6] S. Jackson, L. McGough and H. Verlinde, Conformal bootstrap, universality and gravitational scattering, Nucl. Phys. B 901 (2015) 382 [arXiv:1412.5205] [INSPIRE].

[7] M. Bañados, C. Teitelboim and J. Zanelli, The black hole in three-dimensional space-time, Phys. Rev. Lett. 69 (1992) 1849 [hep-th/9204099] [INSPIRE].

[8] A. Neitzke, Greybody factors at large imaginary frequencies, hep-th/0304080 [INSPIRE].

[9] L. Motl and A. Neitzke, Asymptotic black hole quasinormal frequencies, Adv. Theor. Math. Phys. 7 (2003) 307 [hep-th/0301173] [INSPIRE].

[10] F. Novaes and B. Carneiro da Cunha, Isomonodromy, Painlevé transcendents and scattering off of black holes, JHEP 07 (2014) 132 [arXiv: 1404.5188] [INSPIRE].

[11] B. Carneiro da Cunha and F. Novaes, Kerr-de Sitter greybody factors via isomonodromy, Phys. Rev. D 93 (2016) 024045 [arXiv: 1508.04046] [InSPIRE].

[12] O. Gamayun, N. Iorgov and O. Lisovyy, How instanton combinatorics solves Painlevé VI, V and IIIs, J. Phys. A 46 (2013) 335203 [arXiv:1302.1832] [InSPIRE].

[13] L.F. Alday, D. Gaiotto and Y. Tachikawa, Liouville correlation functions from four-dimensional gauge theories, Lett. Math. Phys. 91 (2010) 167 [arXiv:0906.3219] [INSPIRE].

[14] V.A. Alba, V.A. Fateev, A.V. Litvinov and G.M. Tarnopolskiy, On combinatorial expansion of the conformal blocks arising from AGT conjecture, Lett. Math. Phys. 98 (2011) 33 [arXiv:1012.1312] [INSPIRE].

[15] I. Heemskerk, D. Marolf, J. Polchinski and J. Sully, Bulk and transhorizon measurements in AdS/CFT, JHEP 10 (2012) 165 [arXiv:1201.3664] [INSPIRE].

[16] A. Castro, J.M. Lapan, A. Maloney and M.J. Rodriguez, Black hole monodromy and conformal field theory, Phys. Rev. D 88 (2013) 044003 [arXiv:1303.0759] [InSPIRE].

[17] A. Castro, J.M. Lapan, A. Maloney and M.J. Rodriguez, Black hole scattering from monodromy, Class. Quant. Grav. 30 (2013) 165005 [arXiv: 1304.3781] [INSPIRE].

[18] E. Hijano, P. Kraus, E. Perlmutter and R. Snively, Witten diagrams revisited: the AdS geometry of conformal blocks, JHEP 01 (2016) 146 [arXiv: 1508.00501] [INSPIRE].

[19] A.L. Fitzpatrick, J. Kaplan and M.T. Walters, Virasoro conformal blocks and thermality from classical background fields, JHEP 11 (2015) 200 [arXiv:1501.05315] [INSPIRE]. 
[20] B. Carneiro da Cunha and M. Guica, Exploring the BTZ bulk with boundary conformal blocks, arXiv: 1604.07383 [INSPIRE].

[21] B. Czech, L. Lamprou, S. McCandlish, B. Mosk and J. Sully, A stereoscopic look into the bulk, JHEP 07 (2016) 129 [arXiv: 1604.03110] [INSPIRE].

[22] J. de Boer, F.M. Haehl, M.P. Heller and R.C. Myers, Entanglement, holography and causal diamonds, JHEP 08 (2016) 162 [arXiv:1606.03307] [INSPIRE].

[23] S. Ferrara, A.F. Grillo and R. Gatto, Manifestly conformal covariant operator-product expansion, Lett. Nuovo Cim. 2S2 (1971) 1363 [INSPIRE].

[24] S. Ferrara, A.F. Grillo, G. Parisi and R. Gatto, Canonical scaling and conformal invariance, Phys. Lett. 38B (1972) 333 [INSPIRE].

[25] S. Ferrara, A.F. Grillo, G. Parisi and R. Gatto, Covariant expansion of the conformal four-point function, Nucl. Phys. B 49 (1972) 77 [Erratum ibid. B 53 (1973) 643] [INSPIRE].

[26] A. Castro, A. Maloney and A. Strominger, Hidden conformal symmetry of the Kerr black hole, Phys. Rev. D 82 (2010) 024008 [arXiv: 1004.0996] [inSPIRE].

[27] D. Simmons-Duffin, Projectors, shadows and conformal blocks, JHEP 04 (2014) 146 [arXiv:1204.3894] [INSPIRE].

[28] A.L. Fitzpatrick, J. Kaplan, E. Katz and L. Randall, Decoupling of high dimension operators from the low energy sector in holographic models, arXiv:1304.3458 [INSPIRE].

[29] M. Bochicchio, An asymptotic solution of large- $N$ QCD and of large- $N \mathcal{N}=1 S U S Y Y M$, arXiv: 1409.5149 [INSPIRE].

[30] S.W. Hawking, C.J. Hunter and M. Taylor, Rotation and the AdS/CFT correspondence, Phys. Rev. D 59 (1999) 064005 [hep-th/9811056] [INSPIRE].

[31] H. Lü, J. Mei and C.N. Pope, Kerr/CFT correspondence in diverse dimensions, JHEP 04 (2009) 054 [arXiv: 0811.2225] [inSPIRE].

[32] M. Guica, T. Hartman, W. Song and A. Strominger, The Kerr/CFT correspondence, Phys. Rev. D 80 (2009) 124008 [arXiv:0809.4266] [INSPIRE].

[33] G.W. Gibbons, M.J. Perry and C.N. Pope, The first law of thermodynamics for Kerr-anti-de Sitter black holes, Class. Quant. Grav. 22 (2005) 1503 [hep-th/0408217] [INSPIRE].

[34] H.K. Kunduri and J. Lucietti, Integrability and the Kerr-(A)dS black hole in five dimensions, Phys. Rev. D 71 (2005) 104021 [hep-th/0502124] [INSPIRE].

[35] A.N. Aliev and O. Delice, Superradiant instability of five-dimensional rotating charged AdS black holes, Phys. Rev. D 79 (2009) 024013 [arXiv: 0808. 0280] [InSPIRE].

[36] K. Iwasaki, H. Kimura, S. Shimomura and M. Yoshida, From Gauss to Painlevé: a modern theory of special functions, Aspects of Mathematics E volume 16, Braunschweig, Germany (1991).

[37] M. Jimbo, Monodromy problem and the boundary condition for some Painlevé equations, Publ. Res. Inst. Math. Sci. 18 (1982) 1137.

[38] O. Gamayun, N. Iorgov and O. Lisovyy, Conformal field theory of Painlevé VI, JHEP 10 (2012) 038 [Erratum ibid. 10 (2012) 183] [arXiv:1207.0787] [INSPIRE]. 
[39] A. Litvinov, S. Lukyanov, N. Nekrasov and A. Zamolodchikov, Classical conformal blocks and Painlevè VI, JHEP 07 (2014) 144 [arXiv: 1309.4700] [INSPIRE].

[40] N. Iorgov, O. Lisovyy and J. Teschner, Isomonodromic $\tau$-functions from Liouville conformal blocks, Commun. Math. Phys. 336 (2015) 671 [arXiv:1401.6104] [InSPIRE].

[41] D. Harlow, J. Maltz and E. Witten, Analytic continuation of Liouville theory, JHEP 12 (2011) 071 [arXiv: 1108.4417] [INSPIRE].

[42] P. Di Francesco, P. Mathieu and D. Sénéchal, Conformal field theory, Springer, Germany (1997).

[43] A.B. Zamolodchikov, Two-dimensional conformal symmetry and critical four-spin correlation functions in the Ashkin-Teller model, Zh. Eksp. Teor. Fiz. 90 (1986) 1808.

[44] S. Winitzki, Cosmological particle production and the precision of the WKB approximation, Phys. Rev. D 72 (2005) 104011 [gr-qc/0510001] [INSPIRE].

[45] G. Siopsis, On quasi-normal modes and the AdS $S_{5} / C F T_{4}$ correspondence, Nucl. Phys. B 715 (2005) 483 [hep-th/0407157] [INSPIRE].

[46] M. Bochicchio, Renormalization in large-N QCD is incompatible with open/closed string duality, arXiv:1703.10176 [INSPIRE].

[47] M. Bochicchio, Asymptotic freedom versus open/closed duality in large-N QCD, Phys. Rev. D 95 (2017) 054010 [arXiv: 1606.04546] [INSPIRE]. 